\title{
Simple, exact model of quasisingle field inflation
}

\author{
Yvette Welling $\odot^{*}$ \\ Deutsches Elektronen-Synchrotron DESY, Notkestraße 85, 22607 Hamburg, Germany
}

(Received 10 October 2019; accepted 18 February 2020; published 27 March 2020)

\begin{abstract}
In this paper we present a simple but exact model of quasisingle field inflation [X. Chen and Y. Wang, Quasi-single field inflation and non-Gaussianities, J. Cosmol. Astropart. Phys. 04 (2010) 027, X. Chen and Y. Wang, Large non-Gaussianities with intermediate shapes from quasisingle field inflation, Phys. Rev. D 81, 063511 (2010)], in which the couplings between perturbations are completely controlled, and for instance can be made constant with any desired value. This provides a way to numerically implement quasisingle field inflation and to test its predictions in various regimes. We find that the numerics agree to high accuracy with the analytical predictions for the shape of the bispectrum, given a fixed overall scale. Also, we find reasonable agreement for the amplitude of the isosceles bispectrum, given a fixed base side.

DOI: 10.1103/PhysRevD.101.063535
\end{abstract}

\section{INTRODUCTION}

Quasisingle field inflation [1,2] is a class of models where the inflaton interacts with other particles (isocurvatons) with mass $m \sim H$. It predicts an interesting shape of primordial non-Gaussianities that is distinguishable from single-field inflation [1-12]. The quasisingle field bispectrum has been constrained by cosmic microwave background $(\mathrm{CMB})$ data $[13,14]$ and provides an important target for future observations (for a recent discussion see [15]). Fields with mass of order Hubble may appear naturally in string embeddings of inflation, describing e.g., physics at the Kaluza-Klein scale [16]. Moreover, masses of order Hubble could arise from radiative corrections to light fields [3,17].

Yet it is difficult to test the predictions of quasisingle field inflation with isocurvature mass $m \lesssim H$ numerically with automated software such as PyTransport $[18,19]$, because one needs to construct a potential that sustains a constantly curved trajectory in field space, while keeping $m$ under control. The centrifugal force displaces the inflaton from the minimum of the potential, leading to order Hubble corrections to the isocurvature mass. This problem was discussed in detail in [20] and related considerations can be found in Appendix B of [6]. In this paper we provide a simple model of quasisingle field inflation in which the couplings between perturbations are controlled, and for instance can be made constant with any desired value.

The main obstacle in these potential-based constructions is that, unlike single-field inflation, the potential $V(\varphi)$ does not reflect the properties of the perturbations, because a curved inflationary trajectory deviates from the potential gradient flow in field space. Instead, we apply the recently developed orbital inflation $[21,22]$ to the quasisingle field

**vette.welling@desy.de regime. Orbital inflation is based on the Hamilton-Jacobi formalism developed in [23-26] and extended to multifield in [27-33] in which inflation attracts the Hubble gradient flow

$$
\dot{\phi}^{a} \sim-\nabla^{a} H
$$

This means that the Hubble parameter $H\left(\phi^{a}\right)$ controls the behavior of both the background and perturbations.

The idea of orbital inflation is to align the inflationary trajectory-and thus the Hubble gradient - with an isometry of field space, i.e., an "angular" direction $\theta[21,22]$. This ensures that inflaton happens at constant "radius" $\rho=R$, like in the simplest models of quasisingle field inflation. Additionally, in a moment we will assume a product separable form of $H$ to meet the condition of constant isocurvature mass $m \sim H$. Therefore, the Hubble parameter-as opposed to the potential-takes a particularly simple form to support quasisingle field inflation. Given the existence of an isometry we are free to write the kinetic term in the form

$$
-2 K=f(\rho)(\partial \theta)^{2}+(\partial \rho)^{2} .
$$

Slightly adapting ${ }^{1}$ the multifield Hamilton-Jacobi formalism, the following potentials admit exact inflationary attractors to the angular Hubble gradient flow

\footnotetext{
${ }^{1}$ In the Hamilton-Jacobi formalism there is an additional contribution to the potential of $-2 H_{, \rho}^{2}$. However, on the isometric inflationary attractor this term is zero, and therefore we can preserve the same background solution if we remove this term. As pointed out in [33] the Hamilton-Jacobi assumption bounds the isocurvature mass from above $\mu^{2} \lesssim 3 / 2 H^{2}$, and to study quasisingle field inflation we would like to alleviate this constraint. Therefore, we modified the form of the potential.
} 


$$
V=3 H^{2}-\frac{2 H_{\theta}^{2}}{f(\rho)}
$$

where we denoted $H_{\theta}=\partial_{\theta} H$. Up to the constraint of the vanishing gradient in the radial direction $\partial_{\rho} H=0$, we are free to choose $H(\theta, \rho)$ and we can tune the couplings between perturbations to our liking. This is, to our knowledge, the first exact realization of quasisingle field inflation.

\section{THE MODEL}

Summarizing the discussion above, our simple exact model of quasisingle field inflation is given by

$$
\begin{aligned}
S_{\phi}= & -\frac{1}{2} \int d^{4} x \sqrt{-g}\left[R+f(\rho)(\partial \theta)^{2}+(\partial \rho)^{2}\right. \\
& \left.+6 H^{2}(\theta, \rho)-\frac{4 H_{\theta}^{2}(\theta, \rho)}{f(\rho)}\right]
\end{aligned}
$$

with $H_{\rho}(R)=0$ for some constant value $R$. Furthermore, $R$ is the Ricci scalar of spacetime and we use the convention $M_{p}=1$.

Given some Hubble parameter $H(\theta, \rho)$ the relevant kinematical and geometrical inflationary quantities of this model are given by

$$
\begin{aligned}
\frac{1}{\kappa}= & \frac{f_{\rho}}{2 f}, \quad \dot{\theta}=-2 \frac{H_{\theta}}{f}, \quad \epsilon=\frac{2 H_{\theta}^{2}}{f H^{2}}, \\
\mathbb{R}= & \frac{2}{\kappa^{2}}-\frac{f_{\rho \rho}}{f}, \quad m^{2}=6 H H_{\rho \rho}-\frac{4 H_{\theta} H_{\theta \rho \rho}}{f}, \\
\frac{V_{\rho \rho \rho}}{H^{2}}= & \frac{6 H_{\rho \rho \rho}}{H}+\epsilon\left(\frac{12(2+\mathbb{R} \kappa)}{\kappa^{3}}+\frac{f_{\rho \rho \rho}}{f}\right) \\
& +\frac{4 H_{\theta}}{f H}\left(\frac{6 H_{\theta \rho \rho}}{H \kappa}-\frac{H_{\theta \rho \rho \rho}}{H}\right) .
\end{aligned}
$$

We use the shorthand notation $f=f(\rho), H=H(\theta, \rho)$ and $H_{\rho}=\partial_{\rho} H$, etc. Here $\kappa$ equals the field radius of curvature, $\epsilon \equiv-\frac{\dot{H}}{H^{2}}$ is the first slow-roll parameter, and $\mathbb{R}$ denotes the Ricci curvature of field space. Finally, $m$ is the effective mass of the radial isocurvature perturbations. All variables are understood to be evaluated at $\rho=R$.

To reproduce the results of [1] we focus on a model of quasisingle field inflation with a constant isocurvature mass in Hubble. As can been seen from Eq. (5b), the simplest way to realize a constant isocurvature mass and $V_{\rho \rho \rho}$ (up to a slow-roll correction) is to take a Hubble parameter of the product separable form
$H(\rho, \theta)=W(\theta)\left(1+\frac{\lambda}{2}(\rho-R)^{2}+\frac{\alpha}{6}(\rho-R)^{3}+\cdots\right)$.

A product separable Hubble parameter yields the following subclass of potentials that admit quasisingle field inflation:

$$
\begin{aligned}
V(\theta, \rho)= & 3\left(W^{2}(\theta)-\frac{2 W_{\theta}^{2}(\theta)}{3 f(\rho)}\right) \\
& \times\left(1+\frac{\lambda}{2}(\rho-R)^{2}+\frac{\alpha}{6}(\rho-R)^{3}+\cdots\right)^{2} .
\end{aligned}
$$

Using Eq. (5), the isocurvature mass is given by $m^{2} / H^{2}=6 \lambda+\mathcal{O}(\epsilon)$. In addition, the higher order coupling that is important for non-Gaussianity becomes $V_{\rho \rho \rho}=6 \alpha+\mathcal{O}(\epsilon)$.

Furthermore, we specialize to a flat field metric $f(\rho)=\rho^{2}$, such that $\kappa=R$ on the inflationary trajectory. Moreover, we take $W \sim \theta$, which corresponds to a quadratic type of potential along the angular direction with slowroll parameter $\epsilon=1 /(2 \Delta N+1)$.

\section{NUMERICAL COMPARISON}

We proceed by numerically checking the predictions of quasisingle field inflation. We use the PyTransport code developed by $[18,19]$ (see also $[20,34])$. In what follows the power spectrum and bispectrum are defined as

$$
\begin{gathered}
\langle\mathcal{R}(\mathbf{k}) \mathcal{R}(\mathbf{p})\rangle^{\prime}=P_{\mathcal{R}}(k)=\frac{2 \pi^{2}}{k^{3}} \Delta_{\mathcal{R}}^{2}(k), \\
\left\langle\mathcal{R}\left(\mathbf{k}_{1}\right) \mathcal{R}\left(\mathbf{k}_{2}\right) \mathcal{R}\left(\mathbf{k}_{3}\right)\right\rangle^{\prime}=B_{\mathcal{R}}\left(k_{1}, k_{2}, k_{3}\right)
\end{gathered}
$$

where the prime denotes that the overall delta function $(2 \pi)^{3} \delta_{D}\left(\sum \mathbf{k}_{i}\right)$ has been removed. The bispectrum is usually expressed as a dimensionless shape function for which we use the following two conventions:

$$
\begin{gathered}
\mathcal{S}_{\mathcal{R}}\left(k_{1}, k_{2}, k_{3}\right)=\frac{k_{1}^{2} k_{2}^{2} k_{3}^{2}}{\left(2 \pi^{2}\right)^{2}} \frac{B_{\mathcal{R}}\left(k_{1}, k_{2}, k_{3}\right)}{\Delta_{\mathcal{R}}^{2}\left(k_{1}\right) \Delta_{\mathcal{R}}^{2}\left(k_{2}\right)+\text { perm }}, \\
f_{N L}\left(k_{1}, k_{2}, k_{3}\right) \equiv \frac{5}{6} \frac{B_{\mathcal{R}}\left(k_{1}, k_{2}, k_{3}\right)}{P_{\mathcal{R}}\left(k_{1}\right) P_{\mathcal{R}}\left(k_{2}\right)+\text { perm }} .
\end{gathered}
$$

An analytical expression for the power spectrum for orbital inflation-and hence of the model under consideration-was derived in [22]. The power spectrum is of the form

$$
\Delta_{\mathcal{R}}^{2}=\frac{H^{2}}{8 \pi^{2} \epsilon}(1+\mathcal{D})
$$




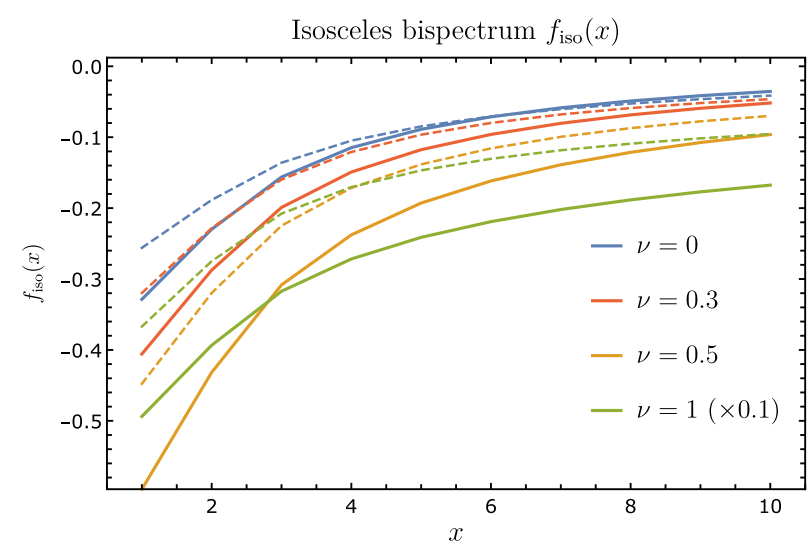

FIG. 1. This figure shows the analytical prediction $f_{\text {ansatz }}^{\mathrm{QSF}}(x)$ in Eq. (17) (dashed lines) and the full numerical solution (solid lines) of the isosceles bispectrum $f_{\text {iso }}(x) \equiv f_{\mathrm{NL}}(k, x k, x k)$, using the code [18]. We take $\nu \in\{0.0,0.3,0.5,1.0\}$ and $k$ to cross the horizon about 60 efolds before the end of inflation. The amplitudes of the analytical ansatz and the corresponding numerical solution for $\nu=1$ have been rescaled by a factor 0.1 to fit inside the same plot range. where $\mathcal{D}$ captures the transfer of power from the isocurvature perturbations. Matching this result with the numerical function $\mathcal{C}(\nu)$ defined in Eq. (3.8) of [1], we find

$$
\mathcal{C}(\nu)=\frac{\kappa^{2}}{16 \epsilon} \mathcal{D}=\frac{1}{2}\left(\frac{1-\exp \left(\left(-\frac{3}{2}+\nu\right) \Delta N\right)}{\frac{3}{2}-\nu}\right)^{2}
$$

with $\nu \equiv \sqrt{9 / 4-m^{2} / H^{2}} \approx \sqrt{9 / 4-6 \lambda}$. This function agrees well with the one shown in Fig. 6 of [1] if we take $\Delta N \sim 50-60$. On the other hand, for $R^{2} \lesssim 10^{2}$ we do not find the same prediction for $n_{s}$ as in Eq. (3.11) of [1], because the scale dependence of $C(\nu)$ becomes important.

Next, we compare the analytical predictions of the quasisingle field bispectrum [1] with the numerical solutions of our exact model. Perturbative control of the quasisingle field results requires $\left|\frac{V_{\rho \rho \rho}}{H}\right| \ll\left|\frac{V_{\rho \rho}}{H^{2}}\right|$, which is amply satisfied if $\alpha \ll 1 / H$. The analytical prediction of
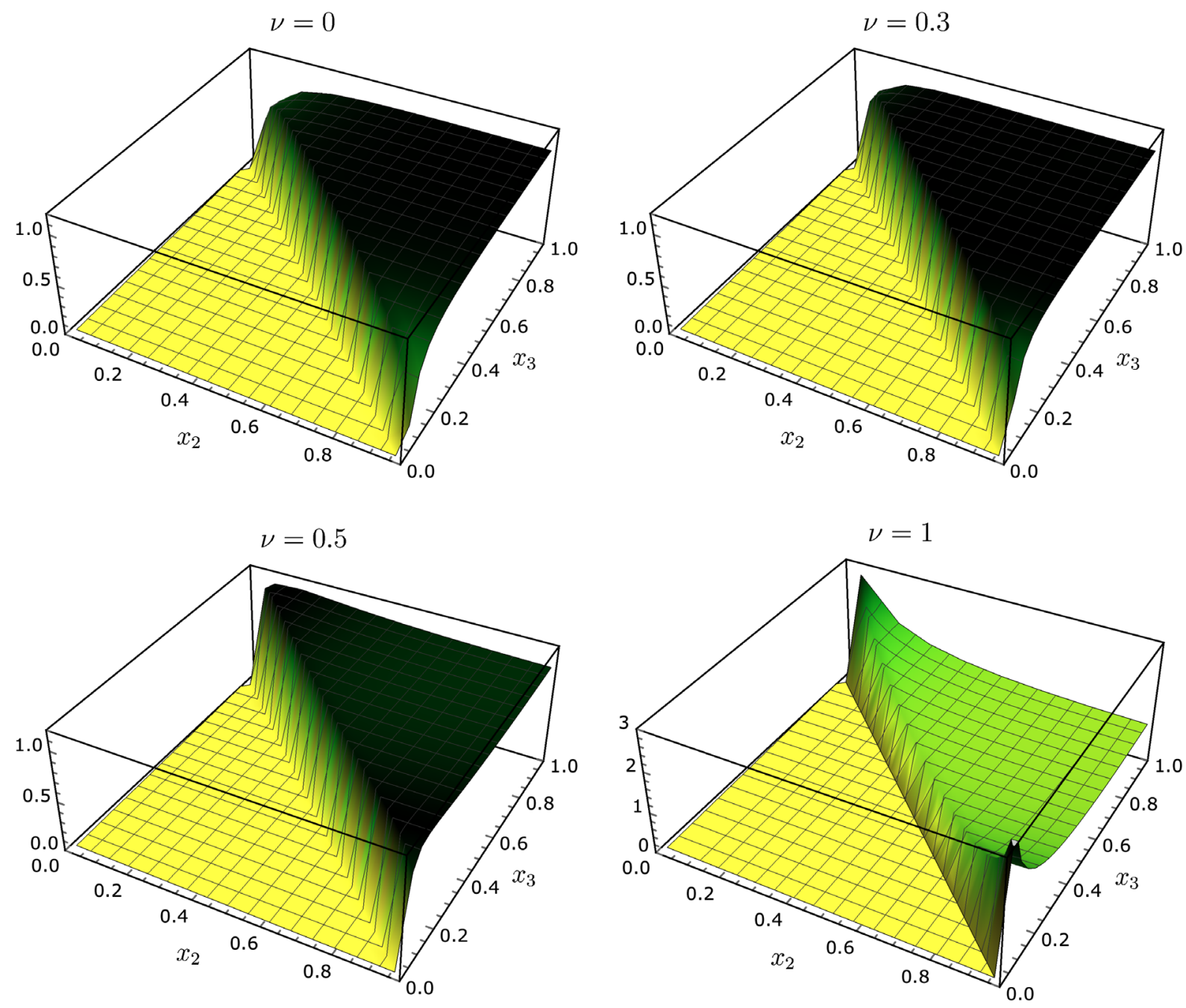

FIG. 2. The numerical solution for the shape function $\mathcal{S}\left(x_{2}, x_{3}\right)$ defined in (18) using the code [18]. We take the values $\nu \in$ $\{0.0,0.3,0.5,1.0\}$ and perform the necessary rescalings, such that this figure can be compared directly to Figure 7 of [1]. 
$f_{\mathrm{NL}}$ in the equilateral configuration [Eq. (4.16) of [1] ] can then be written as

$$
f_{\mathrm{NL}}^{\mathrm{QSF}}=-6 \alpha H \mathcal{A}(\nu)\left(\Delta_{\mathcal{R}}^{2}\right)^{-1 / 2}\left(\frac{2 \epsilon}{R^{2}}\right)^{3 / 2},
$$

with $\mathcal{A}(\nu)$ equal to $\alpha(\nu)$ defined in Eq. (4.16) of [1]. In the regime $\mathcal{D} \ll 1$, that is assumed in [1], we can approximate

$$
f_{\mathrm{NL}}^{\mathrm{QSF}} \approx \mathcal{A}(\nu) \frac{-48 \alpha \pi \epsilon^{2}}{R^{3}}
$$

Notice that $\alpha / R^{3}$ has to be quite large for the amplitude to be non slow-roll suppressed. At the same time we would like to stay in the regime $\mathcal{D} \ll 1$. To compare with the results of [1] we therefore take $\alpha=1000$ and $R=2$.

We start with comparing the shape function for isosceles configurations

$$
f_{\text {iso }}(x) \equiv f_{\mathrm{NL}}(k, x k, x k)
$$
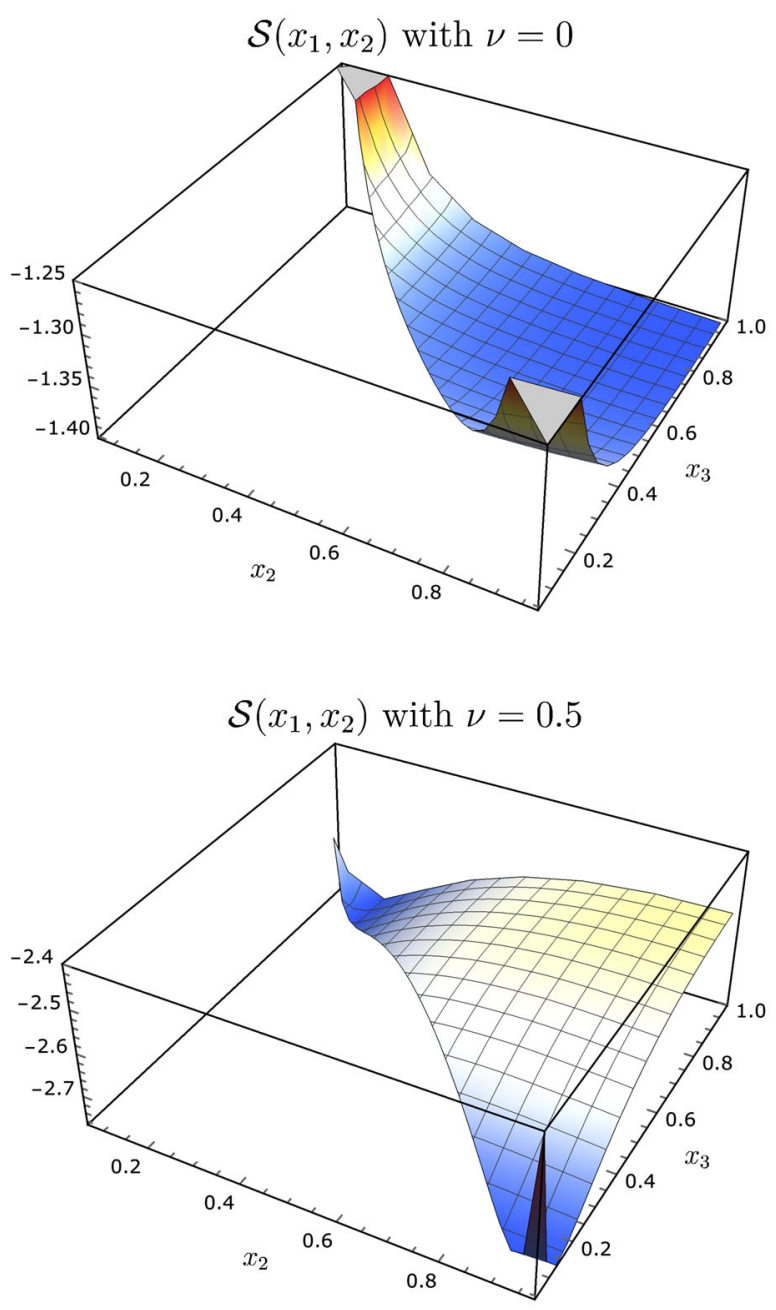

where we suppress the $k$ dependence for convenience. Using the shape ansatz in Eq. (6.1) of [1], the amplitude of the bispectrum for isosceles triangles is given by

$f_{\text {ansatz }}^{\mathrm{QSF}}(x)=\frac{3^{5 / 2} x^{3}}{(1+2 x)^{3 / 2}\left(1+2 x^{3}\right)} \frac{N_{\nu}\left(\frac{8 x^{2}}{(1+2 x)^{3}}\right)}{N_{\nu}\left(\frac{8}{27}\right)} \times f_{\mathrm{NL}}^{\mathrm{QSF}}$,

with $N_{\nu}$ the Neumann function. In the numerical computation we vary $x$ between 1 (equilateral configuration) and $x=10$ (squeezed configuration). Moreover, we take $\nu \in\{0.0,0.3,0.5,1.0\}$ and we take the long wave number to cross the horizon about 60 efolds before the end of inflation. The results are shown in Fig. 1. We see that the overall agreement is reasonably good. The analytical predictions tend to overestimate the amplitude of the bispectrum, except for $\nu=0$ in the squeezed configuration. In the equilateral configuration $x=1$ the analytical prediction for the amplitude is about 1.3 times higher than the full numerical result for all values of $\nu$, whereas in the
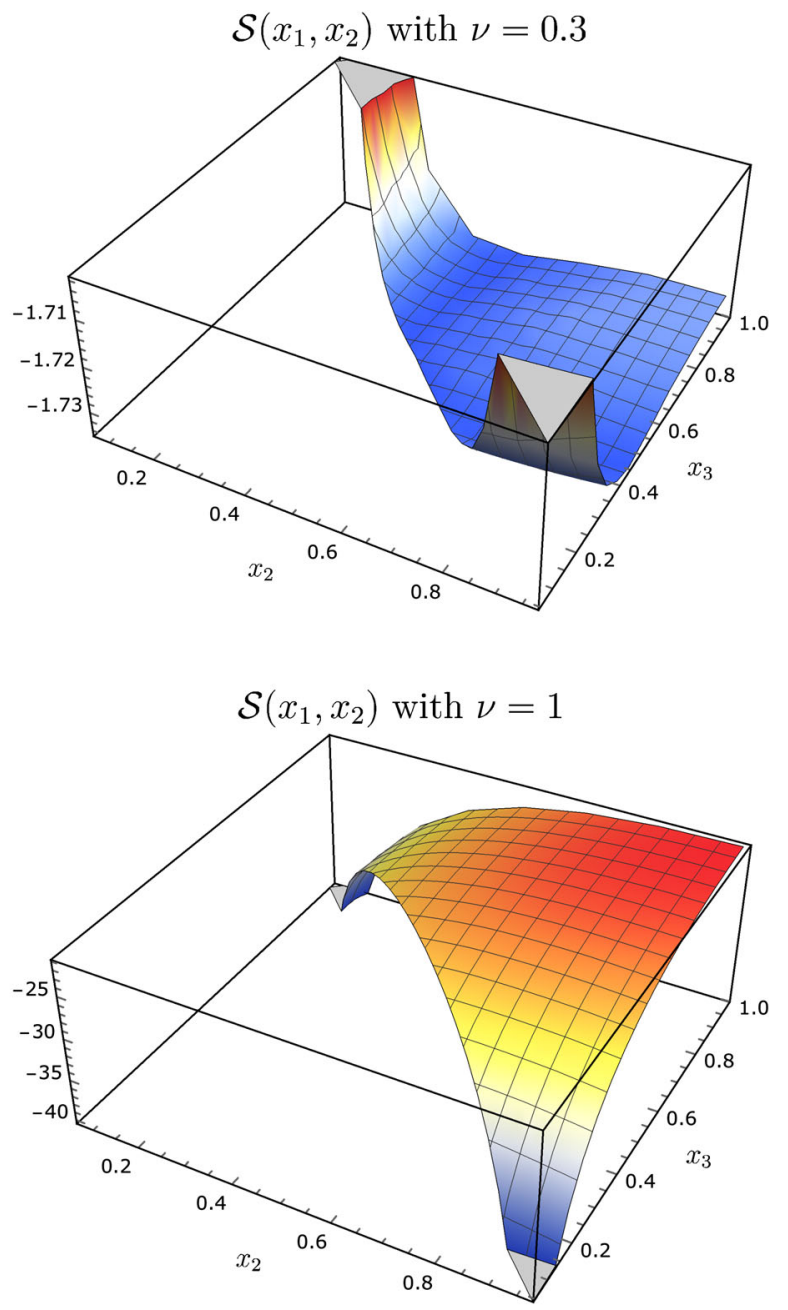

FIG. 3. The same as Fig. 2 except that no rescalings of the shape function have been performed. 
squeezed configuration $x=10$ this ratio ranges from 0.8 to 1.8 .

Next, we compute the shape function

$\mathcal{S}\left(x_{2}, x_{3}\right) \equiv \mathcal{S}_{\mathcal{R}}\left(\frac{k_{t}}{1+x_{2}+x_{3}}, \frac{x_{2} k_{t}}{1+x_{2}+x_{3}}, \frac{x_{3} k_{t}}{1+x_{2}+x_{3}}\right)$,

with the sum of the three wave vectors $k_{t}=k_{1}+k_{2}+k_{3}$ evaluated at 55 efolds before the end of inflation and $x_{2} \in[0,1], \quad x_{3} \in\left[1-x_{2}, 1\right]$. The result for $\nu \in\{0.0,0.3$, $0.5,1.0\}$ is shown in Fig. 2 and for completeness we also show the unscaled shape functions in Fig. 3, in which more detailed shape information can be seen. To compare with Fig. 7 of [1] we normalize the plots in Fig. 2 to one at the equilateral point $\left(x_{2}, x_{3}\right)=(1,1)$. Moreover we take a similar plot range. By eye the shapes are in agreement. To give a more quantitative measure of their correlation we compute the "cosine" [35] between the numerical and analytical shapes. We follow [36] and define the inner product between shapes $F\left(S, S^{\prime}\right)$ that most closely resembles the scaling of the optimal CMB estimator [37] of the amplitude of the bispectrum

$F\left(S, S^{\prime}\right)=\int d^{3} \mathbf{k} w\left(k_{t}\right) S\left(k_{1}, k_{2}, k_{3}\right) S^{\prime}\left(k_{1}, k_{2}, k_{3}\right)$,

with weight function $w\left(k_{t}\right)=1 / k_{t}$. With this definition of the inner product one can define a cosine between shapes

$$
C\left(S, S^{\prime}\right)=\frac{F\left(S, S^{\prime}\right)}{\sqrt{F(S, S) F\left(S^{\prime}, S^{\prime}\right)}} .
$$

The cosine has been shown to provide a good proxy of the CMB cross-correlation between bispectra [36]. The computation of the cosine simplifies considerably, considering the fact that the shape ansatz of quasisingle-field inflation is scale invariant, that is, independent of $k_{t}$

$S_{\nu}^{\mathrm{QSF}}\left(x_{2}, x_{3}\right)=\frac{6}{5} \frac{\left(x_{2} x_{3}\right)^{1 / 2}}{\left(\frac{1+x_{2}+x_{3}}{3}\right)^{3 / 2}} \frac{N_{\nu}\left(\frac{8 x_{x} x_{3}}{\left(1+x_{2}+x_{3}\right)^{3}}\right)}{N_{\nu}\left(\frac{8}{27}\right)} \times f_{\mathrm{NL}}^{\mathrm{QSF}}$,

up to a slight scale dependence in $f_{\mathrm{NL}}^{\mathrm{QSF}}$. Therefore, assuming scale invariance, we may ignore the dependence on the weight function and drop the integral over $k_{t}$ to integrate over $x_{2}$ and $x_{3}$ only, with induced measure $\int d x_{2} d x_{3}\left(1+x_{2}+x_{3}\right)^{-3}$. For all values of $\nu$ we find that the cosine between the quasisingle-field shape ansatz and the numerically computed shape is very close to unity, with a maximal deviation of 0.004 . For comparison, the cosines of the quasisingle-field shape ansatz with the local and equilateral templates [see e.g., Eqs. (35) and (36) in [36] ] take values between 0.73 and 0.88 for the same values of $\nu$.

\section{CONCLUSIONS}

In this paper we provided the first exact model of quasisingle field inflation. With this model we are able to test numerically the analytical predictions of [1] using PyTransport $[18,19]$. In particular, we test for the first time the goodness of the quasisingle field bispectrum shape ansatz [Eq. (6.1) of [1]] for $\nu \in\{0.0,0.3,0.5,1.0\}$.

We find that the numerics agree to high accuracy with the analytical predictions for the shape function, given a fixed overall scale. The "cosine" between the quasisinglefield shape ansatz and the numerically computed shape is in all cases equal or larger than 0.996. Moreover, we find reasonable agreement with the analytical predictions for the amplitude of the isosceles bispectrum, given a fixed base side. The comparison is plotted in Fig. 1. The deviations are configuration and $\nu$ dependent. In the equilateral configuration the analytical prediction for the amplitude is about 1.3 times higher than the full numerical result for all values of $\nu$, whereas in the squeezed configuration this ratio ranges from 0.8 to 1.8 for the different values of $\nu$.

The scale dependence of the bispectrum requires further investigation. The quasisingle field shape ansatz is scale independent. However, similar to what we observe for the power spectrum, a small radius of curvature $R^{2}<10^{2}$ in field space might induce an important contribution to the scale dependence.

\section{ACKNOWLEDGMENTS}

I wish to thank Ana Achúcarro for motivating me to write this paper, and for stimulating discussions. Also, I would like to thank Gonzalo Palma and Dong-Gang Wang for discussions on related work. Y. W. is supported by the ERC Consolidator Grant STRINGFLATION under the HORIZON 2020 Grant Agreement No. 647995.
[1] X. Chen and Y. Wang, Quasi-single field inflation and non-Gaussianities, J. Cosmol. Astropart. Phys. 04 (2010) 027.
[2] X. Chen and Y. Wang, Large non-Gaussianities with intermediate shapes from quasisingle field inflation, Phys. Rev. D 81, 063511 (2010). 
[3] D. Baumann and D. Green, Signatures of supersymmetry from the early universe, Phys. Rev. D 85, 103520 (2012).

[4] V. Assassi, D. Baumann, and D. Green, On soft limits of inflationary correlation functions, J. Cosmol. Astropart. Phys. 11 (2012) 047.

[5] T. Noumi, M. Yamaguchi, and D. Yokoyama, Effective field theory approach to quasi-single field inflation and effects of heavy fields, J. High Energy Phys. 06 (2013) 051.

[6] V. Assassi, D. Baumann, D. Green, and L. McAllister, Planck-suppressed operators, J. Cosmol. Astropart. Phys. 01 (2014) 033.

[7] X. Chen, M. H. Namjoo, and Y. Wang, Models of the primordial standard clock, J. Cosmol. Astropart. Phys. 02 (2015) 027.

[8] N. Arkani-Hamed and J. Maldacena, Cosmological collider physics, arXiv:1503.08043.

[9] H. Lee, D. Baumann, and G. L. Pimentel, Non-Gaussianity as a particle detector, J. High Energy Phys. 12 (2016) 040.

[10] H. An, M. McAneny, A. K. Ridgway, and M. B. Wise, Quasi single field inflation in the non-perturbative regime, J. High Energy Phys. 06 (2018) 105.

[11] N. Arkani-Hamed, D. Baumann, H. Lee, and G. L. Pimentel, The cosmological bootstrap: Inflationary correlators from symmetries and singularities, arXiv:1811.00024.

[12] M. McAneny and A. K. Ridgway, New shapes of primordial non-Gaussianity from quasisingle field inflation with multiple isocurvatons, Phys. Rev. D 100, 043534 (2019).

[13] Y. Akrami et al., Planck 2018 results. X. Constraints on inflation, Astrophys. Space Sci. 364, 69 (2019).

[14] Y. Akrami et al., Planck 2018 results. IX. Constraints on primordial non-Gaussianity, arXiv:1905.05697.

[15] P. D. Meerburg, M. Mnchmeyer, J. B. Muoz, and X. Chen, Prospects for cosmological collider physics, J. Cosmol. Astropart. Phys. 03 (2017) 050.

[16] D. Baumann and L. McAllister, Inflation and String Theory, Cambridge Monographs on Mathematical Physics (Cambridge University Press, Cambridge, England, 2015).

[17] E. J. Copeland, A. R. Liddle, D. H. Lyth, E. D. Stewart, and D. Wands, False vacuum inflation with Einstein gravity, Phys. Rev. D 49, 6410 (1994).

[18] D. J. Mulryne and J. W. Ronayne, PyTransport: A Python package for the calculation of inflationary correlation functions, J. Open Source Software 3, 494 (2018).

[19] J. W. Ronayne and D. J. Mulryne, Numerically evaluating the bispectrum in curved field-space with PyTransport 2.0, J. Cosmol. Astropart. Phys. 01 (2018) 023.

[20] M. Dias, J. Frazer, D. J. Mulryne, and D. Seery, Numerical evaluation of the bispectrum in multiple field inflation-the transport approach with code, J. Cosmol. Astropart. Phys. 12 (2016) 033.
[21] A. Achúcarro, E. J. Copeland, O. Iarygina, G. A. Palma, D.-G. Wang, and Y. Welling, Shift-symmetric orbital inflation: Single field or multi-field?, arXiv:1901.03657.

[22] A. Achucarro and Y. Welling, Orbital inflation: Inflating along an angular isometry of field space, arXiv:1907 .02020 .

[23] A. G. Muslimov, On the scalar field dynamics in a spatially flat Friedman universe, Classical Quantum Gravity 7, 231 (1990).

[24] D. S. Salopek and J. R. Bond, Nonlinear evolution of long wavelength metric fluctuations in inflationary models, Phys. Rev. D 42, 3936 (1990).

[25] J.E. Lidsey, The scalar field as dynamical variable in inflation, Phys. Lett. B 273, 42 (1991).

[26] E. J. Copeland, E. W. Kolb, A. R. Liddle, and J. E. Lidsey, Reconstructing the inflation potential, in principle and in practice, Phys. Rev. D 48, 2529 (1993).

[27] K. Skenderis and P. K. Townsend, Hidden Supersymmetry of Domain Walls and Cosmologies, Phys. Rev. Lett. 96, 191301 (2006).

[28] K. Skenderis and P. K. Townsend, Hamilton-Jacobi method for curved domain walls and cosmologies, Phys. Rev. D 74, 125008 (2006).

[29] C. T. Byrnes and G. Tasinato, Non-Gaussianity beyond slow roll in multi-field inflation, J. Cosmol. Astropart. Phys. 08 (2009) 016.

[30] P. M. Saffin, The covariance of multi-field perturbations, pseudo-susy and $f_{N L}$, J. Cosmol. Astropart. Phys. 09 (2012) 002.

[31] J. Garriga, K. Skenderis, and Y. Urakawa, Multi-field inflation from holography, J. Cosmol. Astropart. Phys. 01 (2015) 028.

[32] J. Garriga, Y. Urakawa, and F. Vernizzi, $\delta N$ formalism from superpotential and holography, J. Cosmol. Astropart. Phys. 02 (2016) 036.

[33] A. Achúcarro, S. Cespedes, A.-C. Davis, and G. A. Palma, Constraints on Holographic Multifield Inflation, Phys. Rev. Lett. 122, 191301 (2019).

[34] D. Mulryne, Data for "PyTransport: A Python package for the calculation of inflationary correlation functions,", Zenodo, https://doi.org/10.5281/zenodo.61239 (2016).

[35] D. Babich, P. Creminelli, and M. Zaldarriaga, The shape of non-Gaussianities, J. Cosmol. Astropart. Phys. 08 (2004) 009.

[36] J. R. Fergusson and E. P. S. Shellard, The shape of primordial non-Gaussianity and the CMB bispectrum, Phys. Rev. D 80, 043510 (2009).

[37] D. Babich, Optimal estimation of non-Gaussianity, Phys. Rev. D 72, 043003 (2005). 